\title{
Obscurin Is Required for the Lateral Alignment of Striated Myofibrils in Zebrafish
}

\author{
Maide Ö. Raeker, ${ }^{1}$ Fengyun Su, ${ }^{2}$ Sarah B. Geisler, ${ }^{1}$ Andrei B. Borisov, ${ }^{1}$ \\ Aikaterini Kontrogianni-Konstantopoulos, ${ }^{3}$ Susan E. Lyons, ${ }^{2}$ and Mark W. Russell ${ }^{1}$
}

\begin{abstract}
Obscurin/obscurin-MLCK is a giant sarcomere-associated protein with multiple isoforms whose interactions with titin and small ankyrin-1 suggest that it has an important role in myofibril assembly, structural support, and the sarcomeric alignment of the sarcoplasmic reticulum. In this study, we characterized the zebrafish orthologue of obscurin and examined its role in striated myofibril assembly. Zebrafish obscurin was expressed in the somites and central nervous system by 24 hours post-fertilization (hpf) and in the heart by $48 \mathrm{hpf}$. Depletion of obscurin using two independent morpholino antisense oligonucleotides resulted in diminished numbers and marked disarray of skeletal myofibrils, impaired lateral alignment of adjacent myofibrils, disorganization of the sarcoplasmic reticulum, somite segmentation defects, and abnormalities of cardiac structure and function. This is the first demonstration that obscurin is required for vertebrate cardiac and skeletal muscle development. The diminished capacity to generate and organize new myofibrils in response to obscurin depletion suggests that it may have a vital role in the causation of or adaptation to cardiac and skeletal myopathies. Developmental Dynamics 235: 2018-2029, 2006. $\odot 2006$ Wiley-Liss, Inc.
\end{abstract}

Key words: obscurin; zebrafish; myofibrillogenesis; somite; sarcomere; heart; development

Accepted 27 February 2006

\section{INTRODUCTION}

Obscurin/obscurin-MLCK is a recently identified giant protein that, in adult mammals, is predominantly expressed in striated muscle (Young et al., 2001; Bang et al., 2001; Russell et al., 2002). Through alternative splicing and at least two translation initiation and termination sites, multiple obscurin isoforms can be expressed (Russell et al., 2002) (Fig. 1). The most widely studied isoform, identified as obscurin, was originally characterized by Young et al. (2001) and encodes for at least 67 tandemly arranged immu- noglobulin domains as well as 2 fibronectin, a calcium/calmodulin-binding, a Rho guanine nucleotide exchange factor (GEF), and an ankyrin binding (Kontrogianni-Konstantopoulos et al., 2003; Bagnato et al., 2003) domain(s). A second giant isoform encodes for all of these domains except for the ankyrin-binding domain and instead has two tandemly arranged carboxy terminal serinethreonine kinase domains related to myosin light chain kinase (MLCK) (Russell et al., 2002). Obscurin-MLCK appears to be the vertebrate ortho- logue of Unc-89 (Sutter et al., 2004), a $732-\mathrm{kD}$ sarcomeric protein that was first characterized in Caenorhabditis elegans (Benian et al., 1996). In nematodes with a mutation of Unc-89, the myosin thick filaments are highly disorganized and lack an M-band in most mutant alleles (Waterston et al., 1980), suggesting that Unc-89 is required for M-band assembly in invertebrates. Like obscurin, Unc-89 has multiple isoforms, some with MLCKlike domains, which appear to have vital and non-redundant roles in myofibril assembly (Small et al., 2004).

\footnotetext{
${ }^{1}$ Department of Pediatrics and Communicable Diseases, University of Michigan, Ann Arbor, Michigan

${ }^{2}$ Department of Internal Medicine, University of Michigan, Ann Arbor, Michigan

${ }^{3}$ Department of Physiology, University of Maryland School of Medicine, Baltimore, Maryland

Grant sponsor: Muscular Dystrophy Association; Grant sponsor: NIH; Grant number: HL075093; Grant sponsor: University of Michigan Biomedical Research Council.

*Correspondence to: Mark W. Russell, M.D., Associate Professor of Pediatrics and Communicable Diseases, Division of Pediatric Cardiology, University of Michigan, L1242 Women's Hospital/Box 0204, 1500 E. Medical Center Dr., Ann Arbor, MI 48109. E-mail: mruss@umich.edu
}

DOI 10.1002/dvdy.20812

Published online 15 June 2006 in Wiley InterScience (www.interscience.wiley.com). 
In vertebrates, much of the work to date has focused on the functions of the obscurin isoform. In mature myocytes, this isoform localizes to both the $\mathrm{Z}$ band, the lattice that supports the actin thin filament array, and the M band, the center of the myosin thick filament assembly (KontrogianniKonstantopoulos et al., 2003; Bagnato et al., 2003; Borisov et al., 2003). Its expression is increased in animals exposed to aortic constriction and its cellular localization is concentrated at sites of new myofibril assembly (Borisov et al., 2003). Based on these studies and its similarity to Unc-89, obscurin has been proposed to have an important role in the assembly of new myofibrils. Recently, this was supported using in vitro models of cardiac (Borisov et al., 2004, 2006) and skeletal muscle (Kontrogianni-Konstantopoulos et al., 2004) myofibrillogenesis. Inhibition of obscurin expression by small interfering RNA expression (Borisov et al., 2006) or overexpression of part of the COOH-terminus of obscurin (Kontrogianni-Konstantopoulos et al., 2004) resulted in an inability to incorporate myosin into the myofibrillar array. Furthermore, gradual cellular depletion of obscurin was associated with the progressive instability of previously assembled myofibrils and an inability to advance past the initial stages of new myofibril assembly (Borisov et al., 2006).

While in vitro studies have suggested that obscurin plays a vital role in vertebrate striated muscle development, the in vivo effects of gene loss have not been examined. Therefore, in this study, obscurin expression was depleted in developing zebrafish embryos using morpholino antisense inhibition. The zebrafish is an increasingly utilized model in the study of vertebrate cardiac and skeletal muscle development due to the similarities of these processes with those in higher vertebrates and the accessibility of the developing embryo (Yelon, 2001; Costa et al., 2002). Morpholino antisense inhibition of obscurin expression allowed the level of depletion to be varied to reveal a range of developmental defects depending on the level of inhibition. Increasing levels of obscurin inhibition were associated with progressive abnormalities of myofibril assembly and somite archi- tecture. Even low dose morpholino injection resulted in decreased numbers and impaired organization of mature myofibrils, a disruption of somite boundaries, and abnormalities of cardiac structure and function. This study is the first to demonstrate a requirement for obscurin in normal vertebrate striated muscle development in vivo. Furthermore, the findings support an important role for obscurin in patterning higher-order muscle structure and, in coordination with other cytoskeletal elements, in stabilizing cellcell and/or cell-matrix interactions.

\section{RESULTS}

\section{Characterization of Zebrafish Orthologues of Obscurin/ Obscurin-MLCK}

In preparation for the morpholino studies, BLAST sequence similarity (tblastn) searches (http://www.ensembl. org/Multi/blastview? species = Danio rerio), using the human obscurin/obscurin-MLCK amino acid sequence as a probe, were performed to determine the number and composition of the zebrafish orthologues of the obscurin/obscurin-MLCK gene. Putative coding sequences corresponding to obscurin/ obscurin-MLCK were identified and localized to two different contiguous genomic DNA sequences (GenBank ID: CR792456 and GenBank IDs: AL844185 and CR394562) using BLAST similarity search (blastn). The genomic sequences AL844185 and CR394562 were determined to overlap and represent a single contiguous genomic sequence. The two genomic contiguous sequences, CR792456 and AL844185/CR394562, have been mapped to zebrafish chromosomes 24 and 8, respectively (http://www.ensembl.org/Danio_rerio).

The genomic DNA sequences were scanned for putative coding sequences using GENSCAN and composite cDNA sequences were assembled for each. The protein predicted to be encoded by the chromosome 24 sequence was similar in size and composition to the obscurin isoform of obscurin/obscurin-MLCK (Young et al., 2001) and was, therefore, labeled the zebrafish obscurin gene. Like mammalian obscurin, it contains a conserved motif (EETITTVVK) and more carboxy ter- minal sequences that have been shown to mediate binding to ankyrin (Kontrogianni-Konstantopoulos et al., 2003; Bagnato et al., 2003), indicating that ankyrin binding is likely to be an important and conserved function of zebrafish obscurin (Fig. 1C).

The zebrafish orthologue of obscurin/ obscurin-MLCK, which mapped to chromosome 8, is predicted to be similar in size and composition to the giant isoform of obscurin-MLCK and has been labeled obscurin-MLCK to distinguish it from the zebrafish obscurin gene locus. Phylogenetic analysis of the RhoGEF domains of the zebrafish obscurin and obscurin-MLCK genes supports the assertion that these coorthologues duplicated and diverged after evolution of the common ancestor shared with higher vertebrates (Fig. 1B).

\section{Expression of Obscurin and Obscurin-MLCK in Zebrafish Embryos}

Reverse transcriptase primed PCR (RT-PCR) was used to amplify a portion of the $5^{\prime}$ end of the zebrafish obscurin gene from mRNA at multiple stages of development. Expression of obscurin in whole embryo mRNA preparations was noted by $22 \mathrm{hr}$ postfertilization (hpf). The level of expression appeared to steadily increase as development proceeded, reaching a peak at approximately 24-48 hpf, with expression persisting into adulthood (Fig. 2A).

Obscurin mRNA expression was observed diffusely throughout the embryo at a low level by whole mount RNA in situ hybridization using a probe from the $5^{\prime}$ end of the gene (data not shown). A similar RNA expression pattern was noted by Thisse et al. (2004) (direct submission to the ZFin database) with a probe to the ankyrin binding domain at the $3^{\prime}$ end of the zebrafish obscurin transcript (see immunostaining of Zfin accession number CK679022 at http:// zfin.org/cgi-bin/webdriver?MIval= aa-fxallfigures.apg\&OID =ZDB-PUB040907-1\&fxallfig_probe_zdb_id=ZDBEST-041111-135). This pattern of RNA expression is consistent with a strong role of post-transcriptional regulation in determining the tissue abundance of obscurin. Therefore, antibodies recognizing different obscurin and obscurin- 
A

Obscurin

Obsouin

is

Cas tho

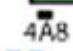

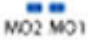

Obscurin-MLCK

Obscurin-tMeC (tandem kinuse iscorm)

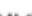

Rho Ank

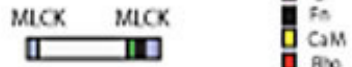

Ano

Obscurin-shCK/single kinre boform

밈

Obsarin gmick

(giark kinase isoform)

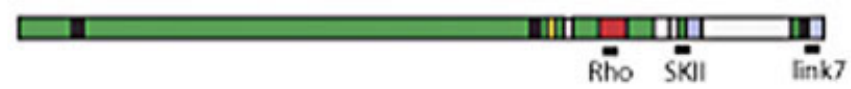

B

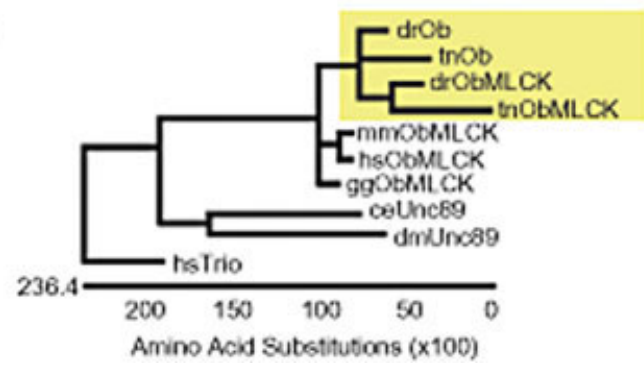

C

drob X K T I I I E E I I T T V V N T R X R H A

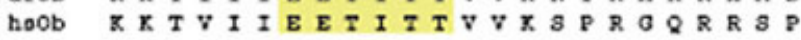

Fig. 1.

A.

Fig. 1. Obscurin/Obscurin-MLCK genes. A: Diagramatic representation of the functional domains of obscurin and obscurinMLCK. Functional domains include immunoglobulin (Ig), fibronectin-3 (Fn), calcium/ calmodulin binding (CaM), RhoGEF (Rho), and MLCK- (MLCK) like motifs. The epitopes recognized by the link7, 4A8, Rho, Ank, and SKII antibodies are indicated. In zebrafish, the obscurin and obscurin-MLCK isoforms are encoded by two different genes while in mammals, they are alternatively spliced products of a single gene. In zebrafish, the smaller obscurin-MLCK isoforms have not yet been identified. B: Phylogenetic tree diagram produced by alignment of the RhoGEF domains from the Unc-89 and obscurin-MLCK family of genes. Included for reference is the corresponding domain from the closely related human Trio protein. Amino acid sequences from the Drosophila melanogaster $(\mathrm{dm})$ and Caenorhabditis elegans (ce) Unc89 proteins and from the zebrafish (dr), pufferfish (tn), chick (gg), mouse (mm), and human (hs) obscurin $(\mathrm{Ob})$ and obscurin-MLCK (ObMLCK) proteins were aligned using the Clustal-W method. Note the close relationship of the corresponding domains from the zebrafish and pufferfish obscurin and obscurin-MLCK genes, supporting duplication and divergence of the obscurin-MLCK gene in ray finned fishes (boxed in yellow). C: Alignment of the ankyrin binding region from the human (hsOb) and zebrafish (drOb) obscurin genes. Note the precise conservation of the putative core binding motif (boxed in yellow).

B.
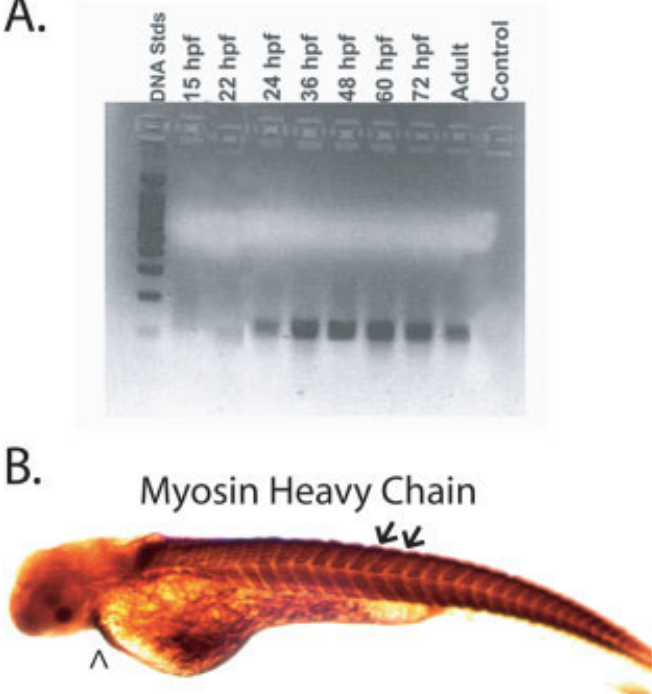

Heart Skeletal Muscle
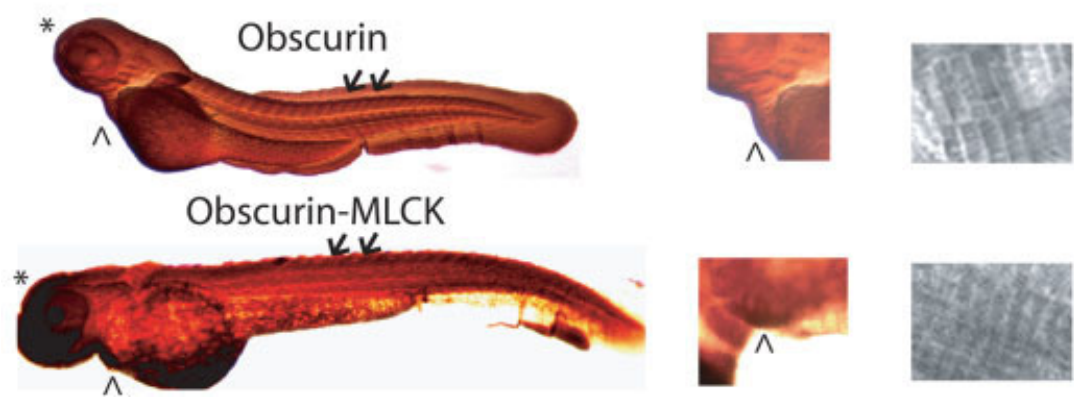

Fig. 2. 
MLCK epitopes were used to examine their tissue and cellular distribution during development. Five antibodies were used in these studies (see Fig. 1A): Rho, a polyclonal antibody that recognizes the RhoGEF domain of both the obscurin and obscurin-gMLCK isoforms; SKII, a polyclonal antibody recognizing the amino MLCK domain of obscurin-MLCK; link7, a polyclonal antibody that recognizes epitopes in the carboxy terminal kinase domain and, therefore, detects all of the human kinase-containing isoforms; Ank, a polyclonal antibody recognizing the ankyrin binding domain of obscurin (Kontrogianni-Konstantopoulos et al., 2003); and 4A8, a monoclonal antibody recognizing an epitope in the amino terminus of obscurin. All antibodies detect the human, mouse, and rat orthologues of obscurin and/or obscurin-MLCK (data not shown). Using whole mount antibody in situ hybridization, each antibody demonstrated nearly identical tissue distribution patterns during development (Fig. 2B and data not shown). Immunostaining for both obscurin (Rho, Ank, and 4A8 antibodies) and obscurin-MLCK (Rho, SKII, and Link7) was noted in the central nervous system and developing somites by 24 hpf. By 48 hpf, expression could also be detected in the heart tube.

The immunolocalization patterns also demonstrated similar but distinct cellular distribution patterns of obscurin and obscurin-MLCK during development. At the onset of myofibril-

Fig. 2. Expression of obscurin and obscurinMLCK in developing zebrafish embryos. A: RTPCR of total RNA from zebrafish embryos using primers from the $5^{\prime}$ end of the obscurin/obscurin-MLCK gene. RNA samples were prepared from whole embryos 15, 22, 24, 36, 48, 60 , and $72 \mathrm{hr}$ post-fertilization (hpf) and from adult zebrafish hearts. Expression is first noted in embryos approximately $22 \mathrm{hpf}$ with expression persisting in the adult heart. B: Immunolocalization of obscurin/obscurin-MLCK expression using the link7 and Ank antibodies in zebrafish embryos at $48 \mathrm{hpf}$. Note the expression of obscurin and obscurin-MLCK in the heart $(\wedge)$ and skeletal muscle (arrows and inset, far right). Immunolocalization of myosin (MF20 antibody), which decorates the A bands of striated muscle, is shown for comparison. Note that, unlike myosin, both obscurin and obscurin-MLCK are also expressed in non-muscle tissues, including the central nervous system $\left.{ }^{*}\right)$, at this stage in development. logenesis, both localized diffusely along newly assembled myofibrils with some organization overlying the $\mathrm{Z}$ and $\mathrm{M}$ bands (Fig. 3A-D). In more mature myofibrils at $72 \mathrm{hpf}$, obscurin became more concentrated at the $\mathrm{M}$ band (Fig. 3E,F) and obscurin-MLCK at the $\mathrm{Z}$ band (Fig. 3G,H). This pattern of sarcomeric localization of obscurin during striated muscle differentiation in zebrafish is consistent with that previously noted in chick cardiac explants (Young et al., 2001), in remodeling rat cardiac myocytes in primary culture (Borisov et al., 2003, 2004) and differentiating C2C12 myoblasts (Kontrogianni-Konstantopoulos et al., 2006). This is the first localization of the vertebrate obscurin-MLCK isoforms during development and is consistent with that noted in rat cardiac myocytes (Russell et al.; unpublished data).

\section{Effect of Obscurin Inhibition on Striated Muscle Development}

The patterns of tissue expression and cellular distribution during development suggest that both zebrafish obscurin and obscurin-MLCK have central roles in vertebrate cardiac and skeletal muscle development. Given the proposed direct involvement of the $\mathrm{COOH}$-terminus of obscurin in myofibril assembly, this isoform was specifically targeted for depletion using antisense morpholino injections. Antisense morpholinos directed at the $5^{\prime}$ untranslated region (MO2) and transcription start site (MO1) of the zebrafish obscurin gene were designed and injected into zebrafish embryos at the 1-4 cell stage. Immunostaining with antibody recognizing the obscurin RhoGEF domain (Rho) demonstrated a reduction in obscurin expression (Fig. 4C). The level of inhibition appeared to be dose dependent with more severely affected embryos demonstrating less obscurin immunostaining than more mildly affected ones using identical reaction conditions (data not shown).

Both morpholinos resulted in very similar phenotypic effects (Fig. 4B). Increasing antisense morpholino dosages were associated with a progressive compromise of striated muscle development, increased curvature and/or growth failure of the tail, increased disruption of the somite boundaries, and more severe cardiac hypoplasia (Fig. 4A,B, and data not shown). At a dose of $6 \mathrm{ng}$ of $\mathrm{MO} 2$ and $12 \mathrm{ng}$ of MO1, 84 and $85 \%$ of injected embryos, respectively, demonstrated irregular somite boundaries and a decreased number of skeletal myofibrils while approximately 54 and 44\%, respectively, also demonstrated cardiac hypoplasia and pericardia edema. Injection of $3 \mathrm{ng}$ of $\mathrm{MO} 2$ was associated with a similar incidence of cardiac and skeletal muscle abnormalities, 55 and $85 \%$, respectively, but diminished severity, including better formed somite boundaries, an increased number of skeletal myofibrils, and less growth impairment relative to the 6-ng dose. Embryo mortality at $72 \mathrm{hpf}$ was $<5 \%$ for the dosages of MO1 used in this study (6 and $12 \mathrm{ng}$ ) and was 0,2 , and $12 \%$ at dosages of 3,6 , and $12 \mathrm{ng}$ of $\mathrm{MO} 2$, respectively. Injection of MOs containing 5 base pair mismatches to MO1 (CMO1: $12 \mathrm{ng}$ ) or MO2 (CMO2: 6 ng) did not result in any morphological defects (Fig. 4B). Due to the specific, consistent abnormalities of striated muscle development and low incidence of embryo mortality observed with the injection of 3 or $6 \mathrm{ng}$ of $\mathrm{MO} 2$, these dosages of MO2 were used in all subsequent studies.

Embryos injected with 3-6 ng of MO2 responded vigorously to initial stimulation although at rest they were somewhat less active than the control injected embryos at all timepoints. Morphant embryos examined at $72 \mathrm{hpf}$ displayed fewer muscle fibers per somite and those myofibrils that did form were disparate and disorganized compared to those in control-injected embryos (Fig. 5A.1,A.2). In many instances, elongated, irregular myofibrils extended beyond the somite boundary, often spanning the length of two somites (Figs. 5A.2, 6). These structures were not noted in any of the control-injected embryos $(\mathrm{N}=12)$ but were noted in all embryos injected with $3 \mathrm{ng}(\mathrm{N}=12)$ or $6 \mathrm{ng}$ $(\mathrm{N}=12)$ of $\mathrm{MO} 2$ that were examined by immunohistochemical or electron microscopic (EM) analysis. In all morphant embryos, $\mathrm{Z}$ band architecture of the individual sarcomeres was intact with normal patterns of $\alpha$-actinin staining and $\mathrm{Z}$ band spacing, despite 
the abnormal myofibril morphology (Fig. 5A.2).

Thick filament organization, how- ever, was not as well preserved (Fig. 5A.4,A.6,B.3). While many myofibrils in the morphant embryos continued to demonstrate a $\mathrm{Z}$ and $\mathrm{M}$ band localization, in general, there was diminished $\mathrm{M}$ band localization of obscurin (Fig.

\section{Obscurin}
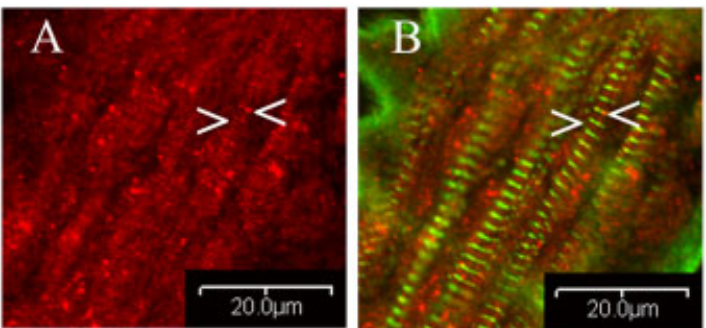

$24 \mathrm{hpf}$

$72 \mathrm{hpf}$
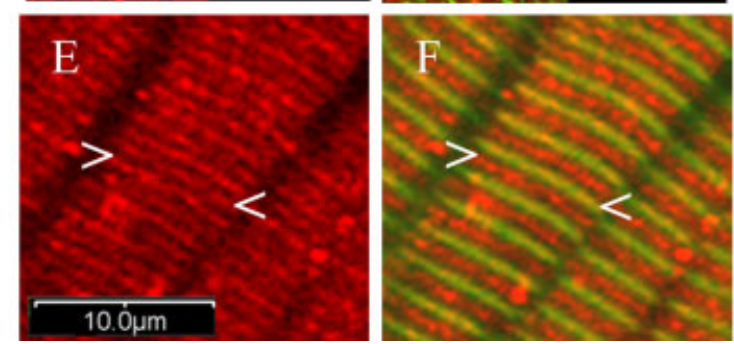

\section{Obscurin-MLCK}
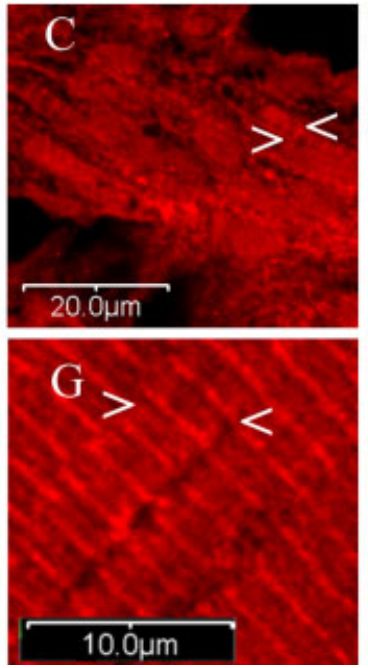
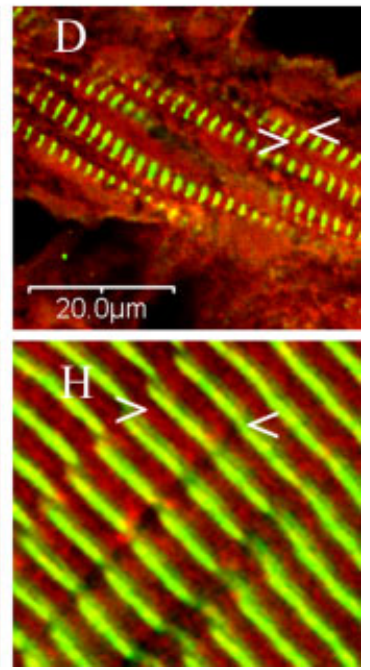

Fig. 3.

A.

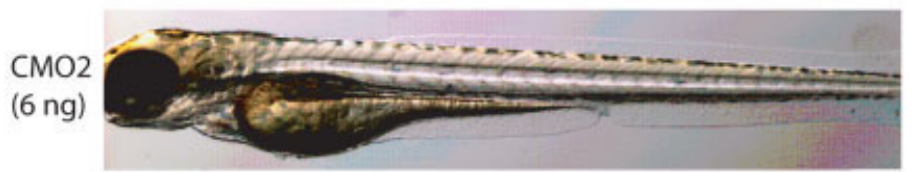

$\mathrm{MO} 2$

(3ng)

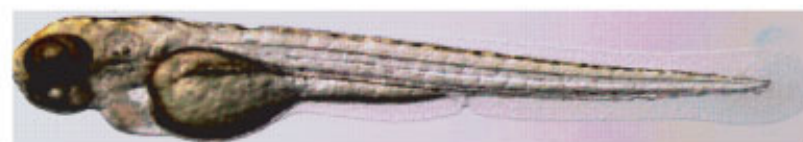

$\mathrm{MO} 2$

(6 ng)

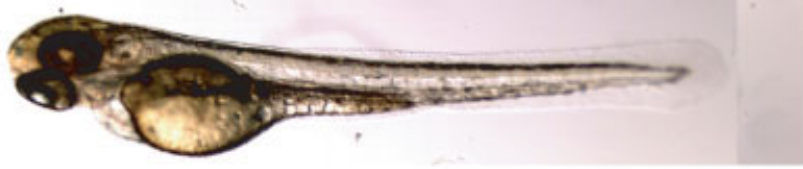

B.

Obscurin Morphant Phenotype

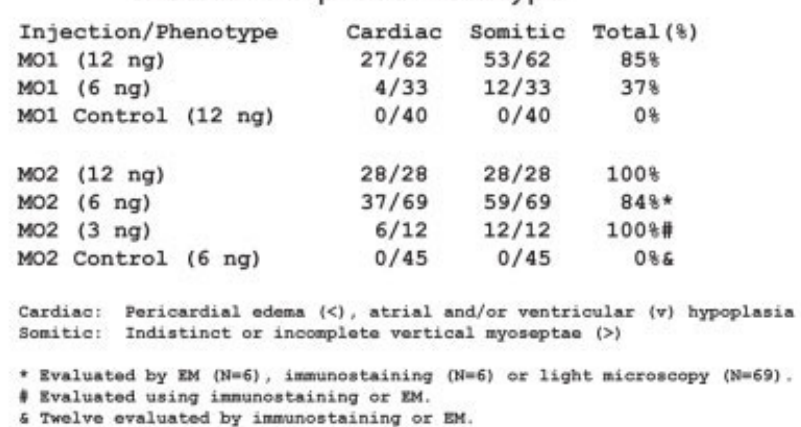

Heart

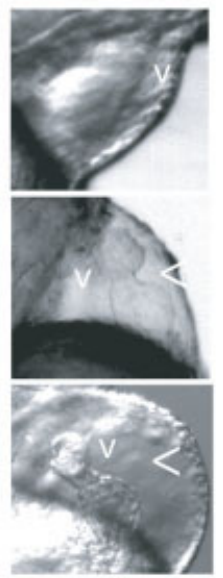

c.
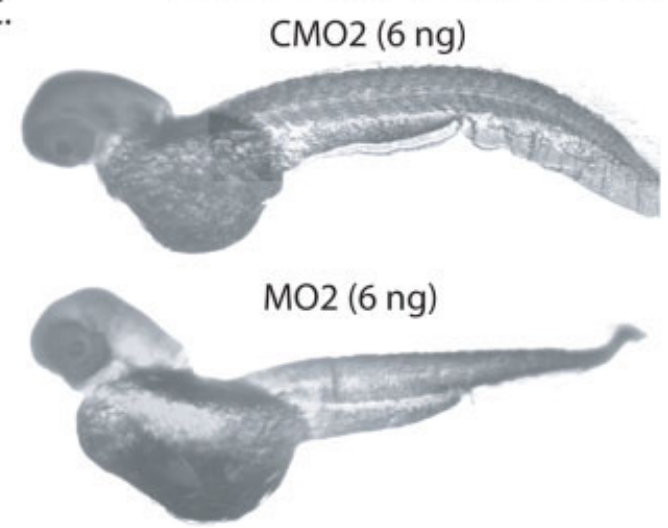

Fig. 4. 
5A.6,A.8) particularly at the 6-ng dose. $\mathrm{M}$ bands were noted to be consistently present by electron microscopy (EM) at the 3-ng dosage (Fig. 5B.2) of MO2 and present in the vast majority of myofibrils even at the 6-ng dosage. However, each embryo injected with $6 \mathrm{ng}$ of $\mathrm{MO} 2$ and examined by EM (6 embryos) displayed occasional abnormalities of thick filament the $\mathrm{A} / \mathrm{I}$ junction, non-parallel thick filament alignment, and the loss of wellformed $\mathrm{M}$ bands (Fig. 5B.3). Both the regional variation in the degree of myofibril organization and obscurin $M$ band localization may be due to regional differences in morpholino concentration and, therefore, in degree of obscurin depletion.

The most dramatic effects of mild obscurin depletion (3 ng dose of MO2) occurred not at the level of the sarcomere but at the integration of striated myofibrils into larger functional units. Specifically, lateral myofibril alignment was impaired in all MO2-injected embryos with the frequency of misaligned adjacent myofibrils increasing with increasing morpholino dose (Fig. 5C,D). In response to 3- or 6-ng MO2 injection, there was noted to be more variable spacing between adjacent myofibrils with an inability to laterally align across the sarcoplasm. These irregularities of lateral alignment were associated with apparent disorganization of the sarcoorganization, including irregularity of plasmic reticulum and disruption of many of the triads, the organized arrangement of T-tubules and junctional SR around the $\mathrm{Z}$ bands in the embryonic skeletal muscle, due to myofibril misalignment.

\section{Effect of Obscurin Inhibition on Somite Development}

One of the more striking effects of obscurin depletion was the dose-dependant disruption of somite boundaries. In zebrafish, somites are separated from each other by the transverse myosepta and divided into ventral and dorsal compartments by the horizontal myoseptum (Holley and NussleinVolhard, 2000). The myosepta are fibrous sheaths that are analogous in structure and function to tendons in that they transmit the force of muscular contractions from skeletal muscle to the bony skeleton to enable movement and locomotion (Holley and Nusslein-Volhard, 2000). In all $(\mathrm{N}=24) 3$ and 6-ng MO2-injected embryos examined by immunostaining or EM, there was noted to be a global disruption of somite segmentation. Transverse myoseptae were irregular and incomplete and only rarely could remnants of the horizontal myoseptum be identified. In response to obscurin depletion, elongated myocytes extended past where somite boundaries should have been. Aberrant terminations of myofibrils were noted mid-somite and there was a marked variability in myofibril length with the majority of myofibrils being longer than expected (Fig. 6A.2). These findings suggest that obscurin is a fundamental component of myoseptal formation, potentially facilitating the organization and anchorage of myofibrils to the extracellular matrix.

\section{Effect of Obscurin Inhibition on Cardiac Development}

The incidence and severity of the cardiac abnormalities was directly correlated with the morpholino dose in the MO1- and MO2-injected embryos. At the 3-ng dose of MO2, there was mild pericardial edema and mild ventricular hypoplasia in $54 \%$ of the embryos examined at $72 \mathrm{hpf}$. At the 6-ng dose of MO2, morphant embryos examined at $72 \mathrm{hpf}$ demonstrated more marked pericardial edema and more severe ventricular hypoplasia than was observed at the 3-ng dose. Irrespective of the occurrence or absence of overt pericardial edema, embryos injected with $6 \mathrm{ng}$ of MO2 had a slower average heart rate than controls [104 \pm 4 beats per minute (bpm) vs. $126 \pm 2$ bpm; $t$-test, $P<0.01]$. As with the skeletal myofibrils, cardiac myofibrils in morphant embryos demonstrated impaired alignment (3 and 6 ng MO2) and thick filament abnormalities $(6 \mathrm{ng}$ MO2) (e.g., "ragged" appearance of the $\mathrm{A} / \mathrm{I}$ junction and indistinct $\mathrm{M}$ bands) (Fig. 7).

\section{DISCUSSION}

Fig. 3. Cellular distribution of obscurin and obscurin-MLCK in zebrafish skeletal muscle during development. Embryos were fixed at 24 (A-D), and 72 (E-H) hpf and co-immunolabeled with antibodies to the ankyrin binding domain of obscurin (Ank) (red: $A, B, E, F)$ or the carboxy terminal kinase domain of obscurin-MLCK (link7) (red: C,D,G,H) and $\alpha$-actinin (green: B,D,F,H). At 24 hpf, while most of the cellular obscurin and obscurin-MLCK remains diffusely localized, some begins to organize around the $Z$ $(A-D:<)$ and $M$ bands $(A-D:>)$ of the maturing myofibrils. It is important to note that all myofibrils with a striated pattern of $\alpha$-actinin staining also demonstrate organization of some of the obscurin and obscurin-MLCK around the M and Z bands. Later in development, by 72 hpf, both obscurin and obscurin-MLCK demonstrate a more distinct striated pattern with obscurin more concentrated at the $M$ bands $(E-H:>)$ and obscurin-MLCK at the $\mathrm{Z}$ bands $(\mathrm{E}-\mathrm{H}:<)$. Similar results were obtained using antibodies to the amino terminal immunoglobulin domains of obscurin (4A8) and the internal kinase domain of obscurin-MLCK (SKII). Scale bars $=20(A-D)$ and $10(E-H) \mu m$.

Fig. 4. Suppression of obscurin expression in MO2-treated embryos. A: Embryos injected with MO2 (MO2; 3 and $6 \mathrm{ng}$ ) or a control morpholino (CMO2; $6 \mathrm{ng}$ ) were examined at 72 hpf. Note that the morphant embryos are slightly shorter with diminished regularity and definition of the transverse myoseptae $(>)$ and moderate pericardial edema $(<)$. Most 72-hpf MO2-treated embryos demonstrated a looped but hypoplastic heart with a small, underdeveloped ventricular (v) chamber compared to control. B: Phenotypic effects of injection with $\mathrm{MO1}$, MO2, or the corresponding control morpholinos, CMO1 and CMO2. Embryos were phenotyped at 72 hpf and categorized as having cardiac and/or somite defects. All embryos injected with control morpholinos at these dosages were morphologically normal. C: Suppression of obscurin expression in MO2-injected embryos (6-ng dose). MO2-injected 72-hpf embryos demonstrate reduced immunostaining for obscurin (Rho $\mathrm{Ab}$ ) compared to controls using identical reaction conditions.

\section{Phylogeny}

Identification of two zebrafish co-orthologues of the mammalian obscurin/obscurin-MLCK gene was not unexpected given the proposed genome duplication event that occurred in teleost fish after evolution of their common ancestor with higher vertebrates (Amores et al., 1998). After duplication, there is often divergent evolution of the two zebrafish co-orthologues such that they acquire unique functions or differing patterns of expression (Postlethwait et al., 2004). This appears to be the case for the zebrafish obscurin and obscurin-MLCK genes. In higher vertebrates and in $C$. el- 
Fig. 5. Obscurin depletion is associated with abnormalities of thick filament assembly and myofibril alignment in zebrafish embryos. At 72 hpf, embryos that had been injected with control (CMO2) or obscurin morpholino (MO2) were evaluated by immunohistochemical (A) and electron microscopic analysis (B). A: Control [CMO2 (6 ng); A.1,3,5] and obscurin morphant [MO2 (3 ng); A.2, (6 ng) A.4,6] embryos were hybridized with antibodies to $\alpha$-actinin (A.12,5-6), and/or obscurin (Ank; A.3-6). A.5 and A.6 correspond to A.3 and A.4. Note that there irregularities of myofibril alignment and orientation in the skeletal muscle of the morphant embryos. The morphant embryos demonstrate a normal $Z$ band structure and spacing as demonstrated by $\alpha$-actinin localization, but there is decreased relative accumulation of obscurin at the $\mathrm{M}$ band $(<)$ as opposed to the $Z$ band $(>)$ (compare A.4 and A.6 to A.3 and A.5). Scale bars $=20 \mu \mathrm{m}$. B: Electron micrographs of skeletal muscle from control [CMO2 (6 ng); B.1] and obscurin morphant [3 $\mathrm{ng}$ (B.2) and $6 \mathrm{ng}$ (B.3) of MO2] embryos. Note the irregular spacing and misalignment of adjacent myofibrils in the morphant embryos. The $Z$ bands $(>)$ of adjacent myofibrils do not align and the sarcoplasmic reticulum $\left(^{*}\right)$ appears disorganized, often lacking the well-ordered triads $(\wedge)$ evident in the control embryos. At the higher morpholino dose, there was occasional disorganization of the thick filaments $(+)$ with irregularity of the $M$ bands $(<)$. C: Myofibril misalignment in control and morphant embryos. The percentage of myofibrils that were not aligned in register with the adjacent myofibril within the same myocyte was significantly greater in morphant than control embryos ( ${ }^{*} t$-test; $P<0.01$ ) and increased with increasing morpholino dose. D: Areas of myofibril disarray were clustered with some areas demonstrating high rates of misalignment. Note the staircase appearance of the $Z$ bands $(>)$ in the skeletal muscle of this 72-hpf embryo injected with $6 \mathrm{ng}$ of $\mathrm{MO} 2$.
A.
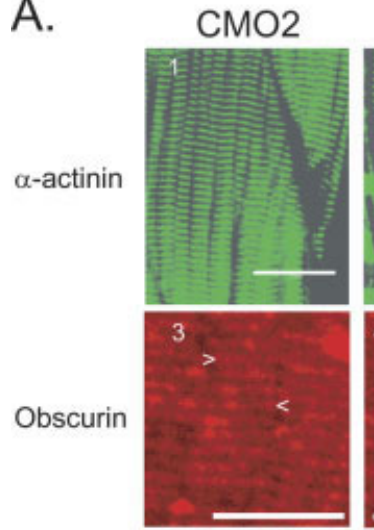

Obscurin/

$\alpha$-actinin

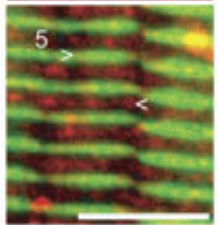

$\mathrm{MO} 2$
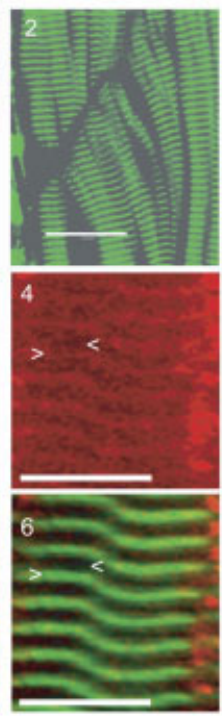

C.

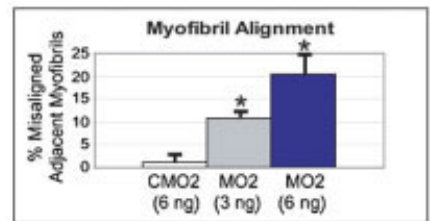

D.

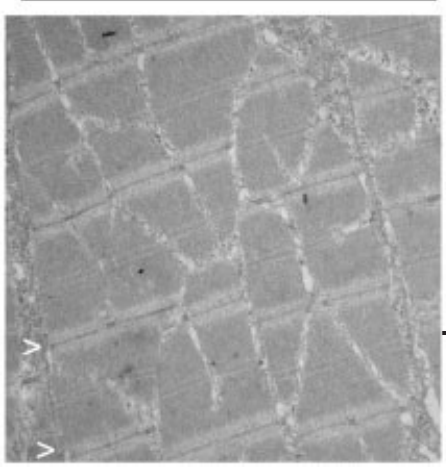

B.
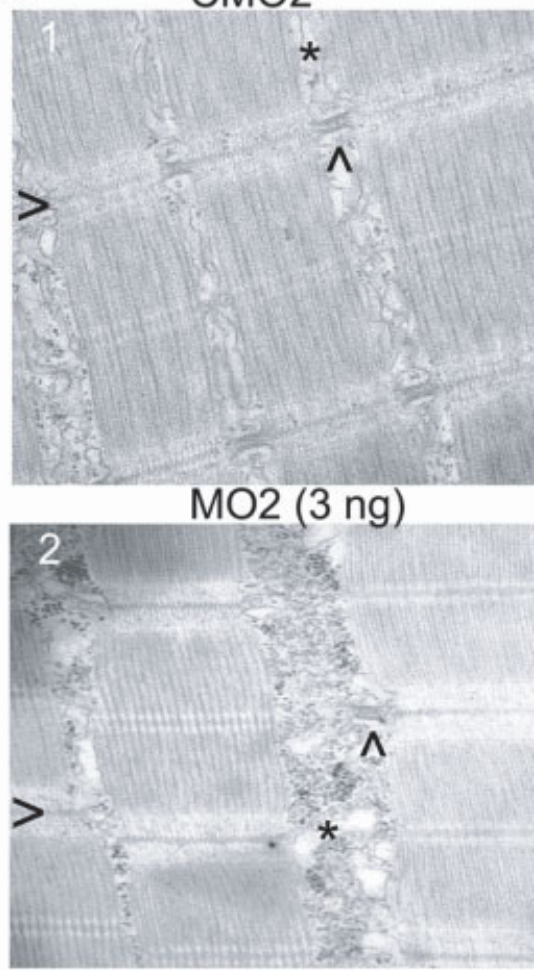

$\mathrm{MO} 2$ (6 ng)

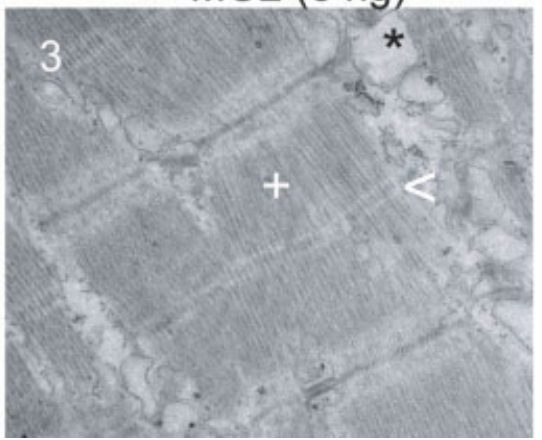

Fig. 5.
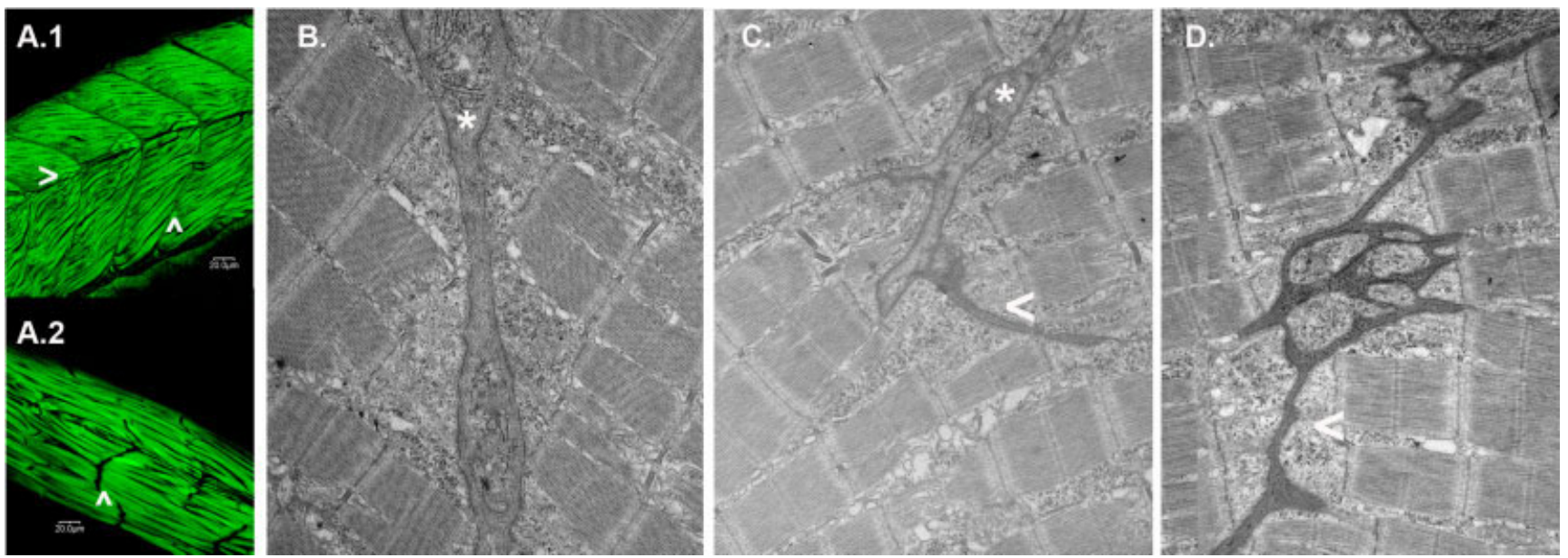

Fig. 6. 

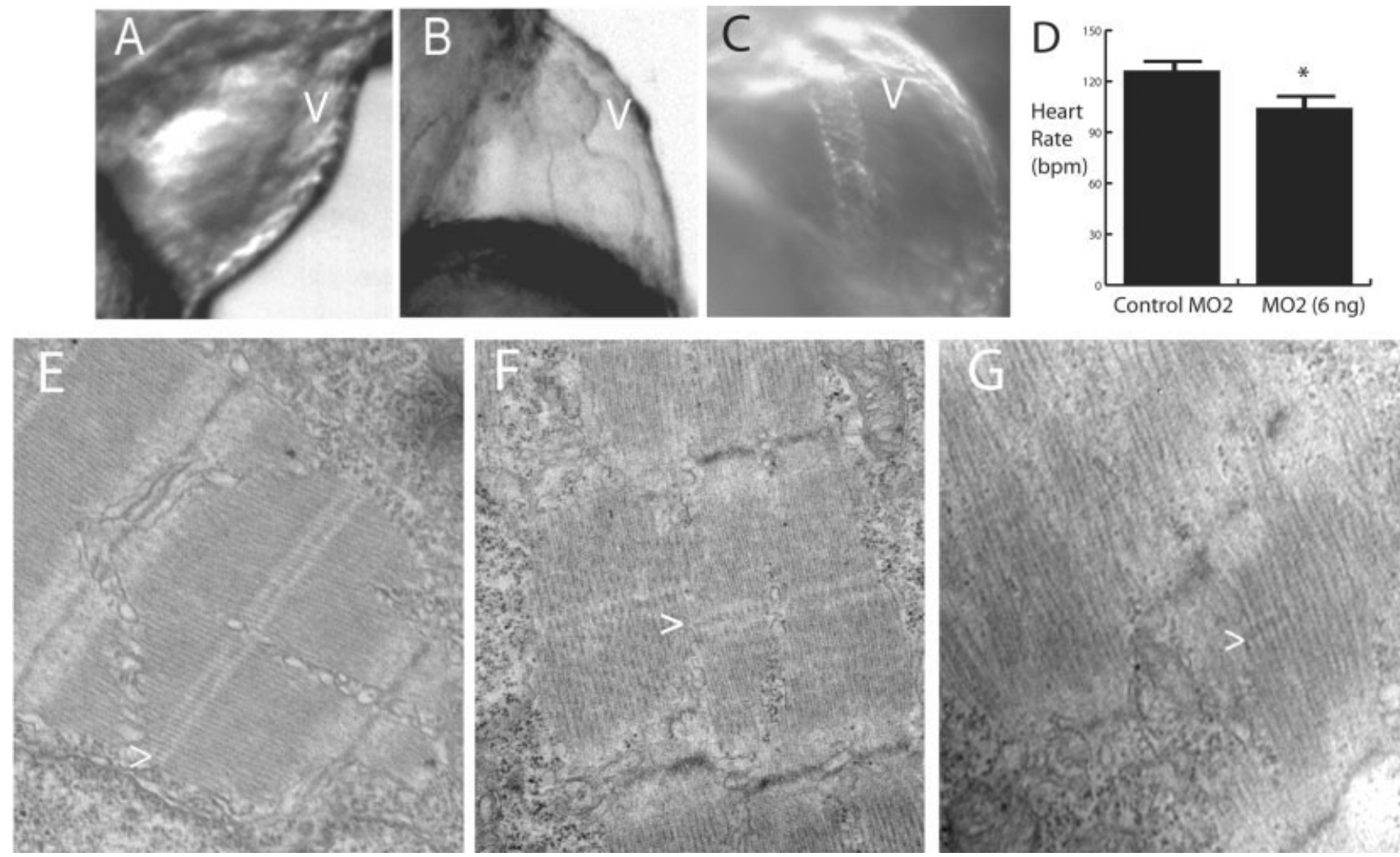

Fig. 7. Cardiac abnormalities in morpholino-treated embryos. A-C: Morphant embryos demonstrated a spectrum of cardiac defects that ranged from mild (B) to severe (C) ventricular (v) hypoplasia. The most severely affected embryos (6 ng of MO2) studied had tube-like hearts (C). A control 72-hpf embryo is shown for comparison (A). All cardiac defects were associated with mild to marked pericardial edema. D: The heart rate of the morphant embryos (6 $\mathrm{ng}$ of MO2) was significantly less than that of the control embryos ( ${ }^{*} t$-test; $P<0.01$ ). As with skeletal muscle, cardiac myofibrils in morphant embryos [3 $\mathrm{ng}(\mathbf{F})$ and $6 \mathrm{ng}(\mathbf{G})$ of $\mathrm{MO}$ ] ] were poorly aligned and organized compared to those in control-injected embryos $(\mathrm{E})$. Some $\mathrm{M}$ bands $(>)$ are apparent in morphant embryos but are much more irregular than in control embryos.

egans, isoforms of obscurin-MLCK and Unc-89 either containing or lacking kinase domains are generated from a single gene (Russell et al., 2002; Small et al., 2004). In zebrafish, the two co-orthologues of obscurin/obscurin-MLCK have evolved such that one expresses only tran-

Fig. 6. Obscurin depletion disrupts somite architecture. A: Embryos injected with $\mathrm{MO} 2$ (6 ng; A.2) or a control morpholino $\mathrm{CMO} 2$ (6 ng; A.1) were fixed and immunostained for $\alpha$-actinin at 72 hpf. Note that in the morphant embryos, there are no detectable horizontal myoseptae $(>)$ and only rudimentary transverse myoseptae $(\wedge)$ compared to control embryos. Elongated, disarrayed myofibrils often extend beyond the length of a normal somite. B-D: Electron micrographs of transverse myoseptae from control [6 ng CMO2 (B)] and obscurin morphant [3 ng (C) and $6 \mathrm{ng}$ (D) of MO2] embryos. Morphant embryos displayed rudimentary transverse myoseptae $\left(C:{ }^{*}, C, D:<\right)$ at the ends of the skeletal myocytes compared to the well-organized transverse myoseptae of control embryos (B: *). scripts lacking the kinase and containing an ankyrin binding motif while the other expresses the kinase domains without the ankyrin binding motif.

The predicted composition of both zebrafish obscurin and obscurinMLCK is highly similar to mammalian obscurin/obscurin-MLCK (Young et al., 2001; Russell et al., 2002) and all of the identified signaling domains and protein interaction sites appear to be conserved in one or both of the zebrafish genes. Furthermore, the zebrafish obscurin and obscurin-MLCK proteins accumulate in a spatially and temporally restricted pattern in the developing embryo that is highly similar to that noted for obscurin/obscurin-MLCK in the rodent (unpublished observations). These similarities between the zebrafish and mammalian obscurin genes strongly support the use of the zebrafish as a model to examine the roles of obscurin during vertebrate development.

\section{Morphant Phenotype}

To examine the role of obscurin in myofibrillogenesis during development, morpholino antisense nucleotides were used to diminish obscurin expression in zebrafish embryos. One of the limitations of using morpholino constructs to examine gene function is differentiating the potential non-specific toxic effects of the morpholino injection from the specific effects of the "knock-down" of target gene expression. This is usually accomplished by demonstrating that the injection of two independent morpholinos causes a similar phenotype that is not apparent in embryos injected with mismatch control morpholinos. Added assurance that the phenotype is specific can be accomplished by demonstrating that the morphant phenotype mimics that of a zebrafish line harboring a mutation of the gene being studied or can be rescued by reintroduc- 
tion of the knocked-down protein. In this study, the morphant phenotype was the same for two independent morpholinos and was not noted in control-injected embryos. However, no mutations of the obscurin gene have been identified in zebrafish and obscurin is too large to express using current approaches. Therefore, validation of the observed morphant phenotype must rely on the consistency of the observations with the previously described in vitro models of obscurin function and with the absence of similar findings in response to non-specific toxic effects of morpholino injection. In previous studies, a number of toxic effects of morpholinos have been reported (reviewed in Heasman, 2002). These include widespread cell death (Braat et al., 2001; Lele et al., 2001), neural degeneration (Nasevicius and Ekker, 2000; Karlen and Rebagliati, 2001), and defects in epiboly (Imai and Talbot, 2001). While these non-specific reactions can affect the development of the notochord resulting in severely misshapen embryos with agenesis or hypoplasia of distal somites, in no instance have defects in somite organization or sarcomere structure been noted. The appearance of these abnormalities even in embryos with relatively mild phenotypic abnormalities (see Fig. 4A.2) suggests that they are specific for the effects of obscurin depletion and do not represent non-specific morpholino toxicity. Furthermore, the findings in this study support and extend those of prior in vitro models of obscurin function as described below.

\section{Obscurin's Role in Myofibril Assembly and Alignment}

Previous studies have proposed that obscurin has important functions in the new myofibril assembly and alignment (Borisov et al., 2003, 2006; Kontrogianni-Konstantopoulos et al., 2004, 2006). The morphant phenotype observed in the injected embryos supports and extends those assertions. The reduction in the number and organization of skeletal myofibrils in response to obscurin treatment indicates that obscurin is required for the assembly of new myofibrils in vivo during development. All myofibrils that did form in the morphant em- bryos contained obscurin suggesting that obscurin is absolutely required for myofibril assembly. That the amount of obscurin per myofibril was not dramatically different in the morphant and control embryos suggests that there may be a minimum number of obscurin molecules required to assemble each sarcomere and that, under normal conditions, obscurin concentration is fairly close to this lower limit. Given the potential energetic cost of producing such a large protein, this is not surprising and is consistent with the observation that obscurin depletion resulted in a reduction in the number of myofibrils rather than a dramatic reduction in the amount of obscurin per myofibril.

However, even a mild reduction in the amount of obscurin per myofibril was sufficient to produce morphologic abnormalities of myofibril structure. Myofibrils that did form were variable in length, often extending past the projected somite boundary, and were not correctly aligned relative to each other. This is consistent with our in vitro studies in which cardiac myocytes completely depleted of obscurin using RNAi-mediated inhibition were unable to generate new myofibrils and those that did form in the presence of limited obscurin displayed marked abnormalities of morphology and alignment (Borisov et al., 2006). Taken together, these studies identify an important scaffolding function of obscurin in the myofibril assembly and alignment process both in vivo and in vitro.

The lateral alignment of adjacent myofibrils is recognized as a critical step in the organization of the sarcoplasmic reticulum, in the structural fixation of the myofibrils, and the subsequent coordinated and efficient transfer of contractile force to the extracellular matrix (reviewed in Bloch et al., 2002). However, the mechanisms directing this alignment have been poorly understood. It was originally thought that desmin and the intermediate filament system might have an important role. While desmin has been demonstrated to participate in the structural support of the myofibril and the maintenance of lateral alignment, mice lacking desmin did not demonstrate widespread abnormalities of myofibril assembly or alignment during development (Milner et al., 1996) suggesting that other structural proteins might initiate the alignment process. This study and our previous work (Borisov et al., 2004, 2006) indicates that obscurin is a critical initial regulator of the lateral alignment and organization of the myofibril, a process that is central to efficient striated muscle function.

There are apparent similarities between the somite and skeletal muscle defects noted in the obscurin morphant embryos in this study and those noted when the expression of the skeletal muscle isoform of titin was inhibited by antisense morpholinos. Although a detailed phenotype of the skeletal muscle titin morphant embryos was not presented, it is interesting that they were also noted to have defects in somite architecture (Xu et al., 2002). Therefore, titin and obscurin may coordinately organize not only the sarcomere and the myofibril but higher-order muscle structures as well, in this case the myotome of the somite. Whether or not other sarcomeric proteins also participate in the organization of the somite is not yet known.

The defects of somite patterning noted in the obscurin morphant embryos are similar to those previously noted in zebrafish embryos depleted of periostin, an extracellular matrix protein proposed to have a role in the establishment of myotendinous junctions (Kudo et al., 2004). The divisions between somites are composed of a sheet of extracellular matrix that includes periostin and other connective tissue proteins such as laminin, fibronectin, tenascin C, and type I collagen (Parsons et al., 2002). This connective tissue appears to form as discontinuous islands of extracellular matrix that coalesce to form a nearly continuous sheath (Henry et al., 2000; Crawford et al., 2003). In zebrafish, a single myocyte spans the length of the somite from one myosepta to the next. In zebrafish depleted of periostin using antisense morpholinos, there was disruption of the myosepta due to variable myofibril length with some myofibrils extending past the projected somite boundary. Furthermore, the number of myofibrils was diminished and the myofibrils that remained were disorganized and varied 
in width (Kudo et al., 2004). This is remarkably similar to the abnormalities noted in the obscurin morphant embryos and suggests an indirect coordination between periostin and obscurin in organizing myofibrillar structure and anchoring the myofibril to the extracellular matrix.

The link between obscurin, an intracellular cytoskeletal protein, and components of the extracellular matrix may involve focal adhesion complexes that have been postulated to mediate somite boundary formation (Henry et al., 2001; Crawford et al., 2003). The invertebrate orthologue of obscurin, Unc-89, has been demonstrated to participate in the formation of integrin adhesion complexes, mediating the organization and attachment of myofilaments to these complexes at the $\mathrm{M}$ band through its interaction with actopaxin (Lin et al., 2003). Recently, it has been demonstrated that organization of fibronectin within the somite boundary by integrin alpha5 is required for proper epithelialization and boundary maintenance (Koshida et al., 2005). It is possible, therefore, that like its invertebrate orthologue, Unc-89, obscurin participates in the linkage of myofibrils to the extracellular matrix through integrin adhesion complexes and that disruption of this linkage affects the stability and organization of the somite boundaries and myofibril architecture.

\section{Parallels to Human Disease}

These studies indicate a critical role of obscurin in new myofibril assembly and alignment in vivo. The parallels between the obscurin and skeletal musclespecific titin morphant embryos support a coordinate function of these two giant proteins in the scaffolding and organization of new myofibrils and the establishment of higher-order muscle structures. Titin and other proteins with similarly critical roles in the assembly and structural support of striated myofibrils including actin, myosin, $\alpha$-actinin, and desmin, have been invariably determined to be responsible for the causation of cardiac and/or skeletal myopathies (Morita et al., 2005). Indeed, the phenotype of the obscurin morphant embryos recapitulates many of the features of myopathic disorders in that, in response to obscurin depletion, there is a marked reduction in the number and organization of muscle fibers.

Given the findings of this study and previous studies, we would anticipate that human mutations of obscurin would also result in a myopathic phenotype. To date, no mutations in the human obscurin gene have been identified. However, multiple myopathies have been mapped to the region on human chromosome 1 (1q42) that encodes for obscurin. While some of these disorders have been determined to be secondary to mutations of cardiac actin, $\alpha$-actinin, or the cardiac ryanodine receptor, all of which map to this "cardiac-rich" region, the genetic cause of at least one 1q42-mapped myopathy, congenital muscular dystrophy type $1 \mathrm{~B}$, has not yet been characterized (Brockington et al., 2000). Given the phenotype of the obscurin morphant embryos in this study, we would anticipate that thorough screening of individuals and families with cardiac or skeletal myopathies would uncover cases attributable to defects in obscurin.

\section{EXPERIMENTAL PROCEDURES}

\section{Zebrafish Maintenance and Breeding}

Zebrafish were maintained and bred and zebrafish embryos were staged as previously described (Westerfield, 1995). Collected embryos were maintained in egg water $(0.006 \%$ Instant Ocean in distilled water) with 2 parts per million methylene blue at $28.5^{\circ} \mathrm{C}$. Embryo age was defined as hours post fertilization (hpf). Experiments were performed using the wild-type EK strain. Embryo medium was supplemented with $0.003 \%$ 1-phenyl-2-thiourea (Sigma, St. Louis, MO) at $24 \mathrm{hpf}$ to prevent pigment formation. Embryos less than $72 \mathrm{hpf}$ were dechorionated with $2 \mathrm{mg} / \mathrm{ml}$ pronase in phosphate-buffered saline (PBS).

\section{Partial Cloning of the Zebrafish Obscurin and Obscurin-MLCK genes}

Cloning of the obscurin-MLCK kinase and Rho guanine nucleotide exchange factor domains were reported previ- ously (Sutter et al., 2004). Additional coding sequence was derived using GENSCAN analysis (http://genes.mit. edu/GENSCAN.html) of the zebrafish genomic DNA sequence to generate PCR primers that were used to amplify the cDNA sequence from adult zebrafish RNA as was described for the cloning of the human obscurin-MLCK (Russell et al., 2002). A partial sequence has been deposited in GenBank (accession number: NM_001003849). The remainder of the proposed zebrafish obscurin-MLCK sequence was determined using BLASTN (http:// www.ncbi.nlm.nih.gov/BLAST) sequence similarity search to construct the genomic DNA sequence encompassing the zebrafish obscurin-MLCK gene. This sequence was searched for putative coding regions using GENSCAN.

During the characterization of the zebrafish obscurin-MLCK gene, a second co-orthologue of obscurin/obscurin-MLCK was identified. The translation initiation codon for the zebrafish obscurin gene was identified by BLAST algorithm (tblastn) sequence homology search of the zebrafish EST database using the corresponding human obscurin amino acid sequence as a probe. The identified zebrafish EST sequences were used to identify the corresponding genomic DNA sequence by BLAST homology search as described above. This genomic DNA sequence was searched for potential coding regions using GENSCAN and a composite obscurin coding sequence constructed.

\section{Phylogenetic Analysis}

Amino acid sequences of the RhoGEF domains from the zebrafish (AY603753.1: AA 1-280), pufferfish (CAG08043: AA 4051-4450), chicken (XP_418501: AA 8428-8800), mouse (BC060226.1: AA 744-1811), and human obscurinMLCK (CAI19285: AA 5697-6008); the zebrafish (AAH91815: AA 214520) and pufferfish (CAF98444: AA 1891-2260) obscurin; and the C. elegans (NP_001020984: AA 153-500) and D. melanogaster (DQ431841: AA 1-280) Unc-89 proteins, were aligned using the Clustal-W alignment algorithm of the DNASTAR program (DNASTAR Inc., Madison, WI). The corresponding domain from the human Trio (AAH60724: AA 826-1200) 
protein was used as a reference for the phylogenetic tree.

\section{Morpholino Oligonucleotide Microinjection}

Obscurin morpholino antisense oligonucleotides targeting the translation initiation site (MO1) and 5'UTR (MO2) or a control morpholino (5 base pair mismatch to MO1 or MO2) were designed by GENE TOOLS, LLC (Philomath, OR). Sequences were as follows: Obscurin antisense MO1, 5'-CCGCCAAATAGATTCTGATCCAT-3'; Control MO1, 5'-CTgCGgCAAATAcATTCTcATCgAT-3'; Obscurin antisense MO2, 5'-TGCTGCTTTCTTTCCCCCCTCAAAT-3'; Control MO2, 5'-TcCTcCTTTgTTTgCCCgCTgAAAT-3' (lowercase letters indicate base changes from the antisense oligos). MOs were dissolved to a stock concentration of 4 $\mathrm{mM}$ in $30 \%$ Danieau Solution, and diluted to $1.5,3$, or $6 \mathrm{ng} / \mathrm{nl}$. Two microliters of diluted morpholino was injected into 1-4-cell-stage embryos using a pneumatic picopump (World Precision Instruments).

\section{RNA Isolation and RT-PCR}

To determine the onset of obscurinMLCK expression, total RNA was isolated from uninjected embryos at 15 , $22,24,36,48,60$, and 72 hpf stages using SV Total RNA Isolation System (Promega, Madison, WI). RNA concentration and purity was determined by optical density reading at $260 \mathrm{~nm}$ and the ratio of $260 / 280 \mathrm{~nm}$ absorbance, respectively. A total of $0.6 \mu \mathrm{g}$ RNA was used in a $25-\mu$ l reaction volume to synthesize cDNA using Access RTPCR kit (Promega Inc.). A fragment of 5'UTR region of zebrafish obscurin cDNA was amplified by PCR (forward primer, 5'-CAGTTCGCGGAATTTACCTG; reverse primer, 5'-TGCTTTCTTTCCTCCCTCAA). As a control, the reaction was performed without AMV reverse transcriptase.

\section{Generation of Obscurin- MLCK Antibodies}

\section{Obscurin-terminal MLCK antibody.}

A 580-bp cDNA encoding for most of the terminal kinase domain of human
obscurin-MLCK was cloned into the pRSET (Invitrogen Corp., Carlsbad, CA) expression vector and the protein expressed in the $E$. coli strain BL21(DE3)pLysS. Induction of expression was carried out at $37^{\circ} \mathrm{C}$ using $0.1 \mathrm{M}$ isopropyl-B-D-thiogalactopyranoside (IPTG). Cells were harvested $5 \mathrm{hr}$ after induction of expression. Expressed protein was purified and dialysed according to Xpress system protein purification protocol (Invitrogen Inc.). The protein was isolated under denaturing conditions and purified using ProBond resin (Invitrogen Inc.). The eluted protein was dialyzed against $10 \mathrm{mM}$ tris, $\mathrm{pH} 8.0,0.1 \%$ Triton-X-100 overnight at $4^{\circ} \mathrm{C}$ to remove urea. The purified protein was supplied to Bethyl Laboratories (Montgomery, TX) for production of polyclonal rabbit antisera. Antibody purification was performed with protein A column (Pierce Inc.) following the manufacturer's instructions.

\section{Obscurin-Rho antibody.}

The Rho antibody was prepared by inserting a 780-bp cDNA fragment encompassing the RhoGEF domain of the human obscurin-MLCK gene into $\mathrm{pR}$ SET vector. Polyclonal antiserum was produced in rabbits using Tris- $\mathrm{HCl}$ gel purified protein (Harlan Bioproducts for Science Inc., Indiannapolis, IN). To affinity purify the Rho antibody, bacterial lysates expressing Rho were loaded onto a Tris- $\mathrm{HCl}$ gel and transferred to nitrocellulose. Ponceau stain was used to visualize the Rho band, which was cut out and washed. Poorly bound protein was removed with $100 \mathrm{mM}$ glycine, $\mathrm{pH}$ 2.5. The nitrocellulose strip was washed and blocked with $3 \%$ bovine serum albumin. Rho polyclonal antiserum was incubated with the strip overnight, and bound antibody was eluted with glycine.

Characterization of the Ank and 4A8 antibodies was described previously by Kontrogianni-Konstantopoulos et al. (2003, 2004), respectively.

\section{Wholemount Immunostaining}

Embryos were fixed for $6 \mathrm{hr}$ with $4 \%$ paraformaldehyde. Single labeling was carried out using Vectastain ABC Elite kit (Vector Laboratories, Burlingame, CA) and the staining was revealed with $\mathrm{DAB}$. The following dilu- tions were used for single staining. MF20 (Developmental Hybridoma Bank, University of Iowa) 1:1, obscurin-Rho 1:2, link7 1:10. Leica MZFLIII dissecting microscope and Nikon microphot-SA compound microscope were used to visualize embryos. For dual labeling, $\alpha$-actinin (Sigma Inc.) (dilution 1:20) in combination with a polyclonal antibody (obscurin-Rho, 1:2; link7, 1:3; obscurin-Ank, 1:10) were used. FITC-conjugated goat antimouse IgG and Texas Red-conjugated Goat Anti-Rabbit IgG (Jackson ImmunoResearch Laboratories) were diluted to 1:100 to reveal staining. For the MF20 antibody, TRITC-conjugated goat anti-mouse IgG (1:100) was used as a secondary antibody. Embryos were mounted onto slides using ProLong Gold Antifade Reagent (Molecular Probes) and visualized on an Olympus FV-500 confocal microscope.

\section{Electron Microscopy}

Embryos at $72 \mathrm{hpf}$ were fixed with $2.5 \%$ glutaraldehyde and $2.0 \%$ paraformaldehyde in $0.1 \mathrm{M}$ Sorenson's buffer for overnight. The embryos were rinsed twice for $15 \mathrm{~min}$ in the buffer and post fixed for $1 \mathrm{hr}$ in $1 \%$ osmium tetroxide in the same buffer. After two 10-min washes with $\mathrm{ddiH}_{2} \mathrm{O}$, they were stained en bloc with saturated uranyl acetate for 30 min. They were then dehydrated with a graded series of ethanol, cleared with propylene oxide, and embedded in Epon 812. Ultra-thin sections were cut on Ultracut $\mathrm{E}$ ultramicrotome (Reichert-Jung, Vienna, Austria) and stained with saturated uranyl acetate and lead citrate. The sections were examined with a Philips CM100 transmission electron microscope, at an accelerating voltage of $60 \mathrm{kV}$. Frequency of myofibril misalignment was scored based on EM images from CMO2(6-ng injection; $\mathrm{N}=3$ ) and MO2-injected (3- and 6-ng injections; $\mathrm{N}=2$ each) embryos. A minimum of 30 adjacent myofibrils were scored for each. Adjacent myofibrils within a given skeletal myocyte from a mid-tail somite were determined to be misaligned if the corresponding $\mathrm{M}$ and $\mathrm{Z}$ bands did not align correctly and the $\mathrm{T}$ tubules were not intact. 


\section{ACKNOWLEDGMENTS}

The authors acknowledge Sasha Meshinchi, Chris Cooke, and Diana Bowen for their assistance with this project and Dr. Robert Bloch and Dr. John Kuwada for helpful discussions and reagents. This work was supported by grants to M.W.R. from the Muscular Dystrophy Association, the NIH (HL075093), and the University of Michigan Biomedical Research Council.

\section{REFERENCES}

Amores A, Force A, Yan YL, Joly L, Amemiya C, Fritz A, Ho RK, Langeland J, Prince V, Wang YL, Westerfield M, Ekker M, Postlethwait JH. 1998. Zebrafish hox clusters and vertebrate genome evolution. Science 282:1711-1714.

Bagnato P, Barone V, Giacomello E, Rossi D, Sorrentino V. 2003. Binding of an ankyrin-1 isoform to obscurin suggests a molecular link between the sarcoplasmic reticulum and myofibrils in striated muscles. J Cell Biol 160:245-253.

Bang ML, Centner T, Fornoff F, Geach AJ, Gotthardt M, McNabb M, Witt CC, Labeit D, Gregorio CC, Granzier H, Labeit S. 2001. The complete gene sequence of titin, expression of an unusual approximately $700-\mathrm{kDa}$ titin isoform, and its interaction with obscurin identify a novel Z-line to I-band linking system. Circ Res 89:1065-1072.

Benian GM, Tinley TL, Tang X, Borodovsky M. 1996. The Caenorhabditis elegans gene unc-89, required fpr muscle M-line assembly, encodes a giant modular protein composed of $\mathrm{Ig}$ and signal transduction domains. J Cell Biol 132:835-848.

Bloch RJ, Capetanaki Y, O'Neill A, Reed P, Williams MW, Resneck WG, Porter NC, Ursitti JA. 2002. Costameres: repeating structures at the sarcolemma of skeletal muscle. Clin Orthop Relat Res 403(Suppl):S203-210.

Borisov AB, Raeker MO, KontrogianniKonstantopoulos A, Yang K, Kurnit DM, Bloch RJ, Russell MW. 2003. Rapid response of cardiac obscurin gene cluster to aortic stenosis: differential activation of Rho-GEF and MLCK and involvement in hypertrophic growth. Biochem Biophys Res Commun 310:910-918.

Borisov AB, Kontrogianni-Konstantopoulos A, Bloch RJ, Westfall MV, Russell MW. 2004. Dynamics of obscurin localization during differentiation and remodeling of cardiac myocytes: obscurin as an integrator of myofibrillar structure. J Histochem Cytochem 52:1117-1127.

Borisov AB, Sutter SB, Kontrogianni-Konstantopoulos A, Bloch RJ, Westfall MV, Russell MW. 2006. Essential role of obscurin in myofibrillogenesis and myofibrillar alignment: evidence from small interfering RNA-mediated gene silencing. Histochem Cell Biol 125:227-238.

Braat AK, van de Water S, Korving J, Zivkovic D. 2001. A zebrafish vasa morphant abolishes vasa protein but does not affect the establishment of the germline. Genesis 30:183-185.

Brockington M, Sewry CA, Herrmann R, Naom I, Dearlove A, Rhodes M, Topaloglu H, Dubowitz V, Voit T, Muntoni F. 2000. Assignment of a form of congenital muscular dystrophy with secondary merosin deficiency to chromosome $1 \mathrm{q} 42$. Am J Hum Genet 66:428-435.

Costa ML, Escaleira RC, Rodrigues VB, Manasfi M, Mermelstein CS. 2002. Some distinctive features of zebrafish myogenesis based on unexpected distributions of the muscle cytoskeletal proteins actin, myosin, desmin, alpha-actinin, troponin and titin. Mech Dev 116:95-104.

Crawford BD, Henry CA, Clason TA, Becker AL, Hille MB. 2003. Activity and distribution of paxillin, focal adhesion kinase, and cadherin indicate cooperative roles during zebrafish morphogenesis. Mol Biol Cell 14:3065-3081.

Heasman J. 2002. Morpholino oligos: making sense of antisense? Dev Biol Mar 15; 243:209-214.

Henry CA, Hall LA, Burr Hille M, SolnicaKrezel L, Cooper MS. 2000. Somites in zebrafish doubly mutant for knypek and trilobite form without internal mesenchymal cells or compaction. Curr Biol 10: 1063-1066.

Henry CA, Crawford BD, Yan YL, Postlethwait J, Cooper MS, Hille MB, 2001. Roles for zebrafish focal adhesion kinase in notochord and somite morphogenesis. Dev Biol 240:474-487.

Holley SA, Nusslein-Volhard C. 2000. Somitogenesis in zebrafish. Curr Top Dev Biol 47:247-277.

Imai Y, Talbot WS. 2001 Morpholino phenocopies of the bmp2b/swirl and bmp7/snailhouse mutations. Genesis 30:160-163.

Karlen S, Rebagliati M. 2001. A morpholino phenocopy of the cyclops mutation. Genesis 30:126-128.

Kontrogianni-Konstantopoulos A, Jones EM, van Rossum DB, Bloch RJ. 2003. Obscurin is a ligand for small ankyrin 1 in skeletal muscle. Mol Biol Cell 14:1138-1148.

Kontrogianni-Konstantopoulos A, Catino DH, Strong JC, Randall WR, Bloch RJ. 2004. Obscurin regulates the organization of myosin into A bands. Am J Physiol Cell Physiol 287:C209-C217.

Kontrogianni-Konstantopoulos A, Catino DH, Strong JC, Bloch RJ. 2006. De novo myofibrillogenesis in $\mathrm{C} 2 \mathrm{C} 12$ cells: evidence for the independent assembly of M-lines and Z-disks. Am J Physiol Cell Physiol 290:C626-637.

Koshida S, Kishimoto Y, Ustumi H, Shimizu T, Furutani-Seiki M, Kondoh H. Takada S. 2005. Integrinalpha5-dependent Fibronectin accumulation for maintenance of somite boundaries in zebrafish embryos. Dev Cell 8:587-598.

Kudo H, Amizuka N, Araki K, Inohaya K, Kudo A. 2004. Zebrafish periostin is required for the adhesion of muscle fiber bundles to the myoseptum and for the differentiation of muscle fibers. Dev Biol 267:473-487.
Lele Z, Bakkers J, Hammerschmidt M. 2001. Morpholino phenocopies of the swirl, snailhouse, somitabun, minifin, silberblick, and pipetail mutations. Genesis 30:190-194.

Lin X, Qadota H, Moerman DG, Williams BD. 2003. C. elegans PAT-6/actopaxin plays a critical role in the assembly of integrin adhesion complexes in vivo. Curr Biol 13:922-932.

Milner DJ, Weitzer G, Tran D, Bradley A, Capetanaki Y. 1996. Disruption of muscle architecture and myocardial degeneration in mice lacking desmin. J Cell Biol 134:1255-1270.

Morita H, Seidman J, Seidman CE. 2005. Genetic causes of human heart failure. J Clin Invest 115:518-526.

Nasevicius A, Ekker SC. 2000. Effective targeted gene 'knockdown' in zebrafish. Nat Genet 26:216-220.

Parsons MJ, Campos I, Hirst EM, Stemple DL. 2002. Removal of dystroglycan causes severe muscular dystrophy in zebrafish embryos. Development 129:3505-3512.

Postlethwait J, Amores A, Cresko W, Singer A, Yan YL. 2004. Subfunction partitioning, the teleost radiation and the annotation of the human genome. Trends Genet 20:481-490.

Russell MW, Raeker MO, Korytkowski KA, Sonneman KJ. 2002. Identification, tissue expression and chromosomal localization of human Obscurin-MLCK, a member of the titin and $\mathrm{Dbl}$ families of myosin light chain kinases. Gene 282:237-246.

Small TM, Gernert KM, Flaherty DB, Mercer KB, Borodovsky M, Benian GM. 2004. Three new isoforms of Caenorhabditis elegans UNC-89 containing MLCKlike protein kinase domains. J Mol Biol 342:91-108.

Sutter SB, Raeker MO, Borisov AB, Russell MW. 2004. Orthologous relationship of obscurin and Unc-89: phylogeny of a novel family of tandem myosin light chain kinases. Dev Genes Evol 214:352-359.

Thisse B, Heyer V, Lux A, Alunni V, Degrave A, Seiliez I, Kirchner J, Parkhill JP, Thisse C. 2004. Spatial and temporal expression of the zebrafish genome by large-scale in situ hybridization screening. Methods Cell Biol 77:505-519.

Waterston RH, Thomson JN, Brenner S, 1980. Mutants with altered muscle structure of Caenorhabditis elegans. Dev Biol 77:271-302.

Westerfield M., 1995. The zebrafish book: a guide for the laboratory use of zebrafish (Danio rerio), 3rd ed. Eugene, OR: University of Oregon Press.

Xu X, Meiler SE, Zhong TP, Mohideen M, Crossley DA, Burggren WW, Fishman MC, 2002. Cardiomyopathy in zebrafish due to mutation in an alternatively spliced exon of titin. Nat Genet 30:205-209.

Yelon D, 2001. Cardiac patterning and morphogenesis in zebrafish. Dev Dyn 222 : 552-563.

Young P, Ehler E, Gautel M, 2001. Obscurin, a giant sarcomeric Rho guanine nucleotide exchange factor protein involved in sarcomere assembly. $\mathrm{J}$ Cell Biol 154:123-136. 